\title{
Avaliação da satisfação com a assistência odontológica na perspectiva de usuários brasileiros adultos: análise multinível
}

\author{
Evaluation of satisfaction with dental care from the standpoint \\ of adult Brazilian users: a multilevel analysis
}

Danilo Lima Carreiro ${ }^{1}$

Renata Francine Rodrigues Oliveira ${ }^{2}$

Wagner Luiz Mineiro Coutinho ${ }^{3}$

Andréa Maria Eleutério de Barros Lima Martins ${ }^{2}$

Desireé Sant'Ana Haikal ${ }^{2}$

\footnotetext{
${ }^{1}$ Departamento de Administração, Instituto Federal do Norte de Minas Gerais. R. Prof. Monteiro Fonseca 216, Vila Brasília. 39400-149 Montes Claros MG Brasil.

coutinhowlm@gmail.com ${ }^{2}$ Departamento de Odontologia, Universidade Estadual de Montes Claros. Montes Claros MG Brasil.

${ }^{3}$ Instituto de Ciências da Saúde, Associação Educativa do Brasil. Montes Claros MG Brasil.
}

\begin{abstract}
The scope of this paper was to evaluate the satisfaction regarding dental care services and to identify the association between dissatisfaction and contextual/individual variables. It involved a cross-sectional study of a representative sample of 8,943 adults from 177 municipalities, in which $14.9 \%$ of adults were dissatisfied. In the multiple analysis there was a greater chance of dissatisfaction with dental services among adults residing in cities with greater social inequality (OR: 1.53, 95\% CI: 1.31-1.81) and with a lower proportion of dentists per inhabitant (OR: 1.17; 95\% CI: 1.00-1.37); yellow/black/brown/indigenous (OR: 1.12; 95\% CI: 0.99-1.27); lower schooling (OR: 1.14; 95\% CI: 0.98-1.33); consultation due to oral problems (OR: 1.23; 95\% CI: 1.04-1.44); (OR: 2.60; 95\% CI: 2.53-3.02) and impact of oral disorders on daily performance (OR: 1.48; 95\% CI: 1.30-1.69). The implementation or adequacy of public policies with the aim of improving satisfaction with dental services should prioritize those municipalities with greater social inequality and with fewer dentists and socially disadvantaged users, who self-perceive oral problems, are dissatisfied with their oral health and suffer impacts resulting from oral problems.
\end{abstract}

Key words Patient satisfaction, Health services, Quality of health care, Public health dentistry, Epidemiology
Resumo Objetivou-se avaliar a satisfação quanto aos serviços de assistência odontológica $e$ identificar associação entre a insatisfação e as variáveis contextuais/individuais. Estudo transversal multinivel de dados secundários de uma amostra representativa de 8.943 adultos de 177 municípios. Encontravam-se insatisfeitos 14,9\% dos adultos. Registrou-se maior chance de insatisfação com os serviços odontológicos entre adultos que residiam em municípios com maior desigualdade social (OR:1,53;IC $\left.{ }_{95 \%}: 1,31-1,81\right) e$ com menor proporção de dentistas por habitante (OR:1,17;IC $\left.{ }_{95 \%}: 1,00-1,37\right)$; amarelos/negros/pardos/indígenas (OR:1,12; IC $\left.{ }_{95 \%}: 0,99-1,27\right)$; menor escolaridade (OR:1,14; IC ${ }_{95 \%}: 0,98-1,33$ ); consulta por motivo de problemas bucais (OR:1,23; $\left.I C_{950}: 1,04-1,44\right)$; insatisfeitos com os dentes e boca (OR:2,60;IC $\left.C_{95 \%}: 2,53-3,02\right)$ e com impacto das desordens bucais no desempenho diário (OR:1,48; IC $\left._{95 \%}: 1,30-1,69\right)$. A implementação ou adequação de políticas públicas com o intuito de melhorar a satisfação com os serviços odontológicos deve priorizar os municípios com maior desigualdade social e com menos dentistas e usuários socialmente desfavorecidos, que autopercebem problemas bucais, insatisfeitos com sua saúde bucal e com impactos decorrentes dos problemas bucais.

Palavras-chave Satisfação do paciente, Serviços de saúde, Qualidade da assistência à saúde, Odontologia em saúde pública, Epidemiologia 


\section{Introdução}

Pesquisas que avaliam os serviços de saúde podem promover, indiretamente, impacto positivo na condição de saúde da população, por meio de seus indicadores. A satisfação com serviços odontológicos pode ser avaliada tanto na perspectiva dos profissionais, gestores e usuários, sendo que a perspectiva dos usuários tem sido bem valorizada ${ }^{1}$, pois pode direcionar tais serviços ao atendimento das demandas da população ${ }^{2}$. Dentre os diversos modos de se avaliar a satisfação com os serviços de saúde, o modelo teórico proposto por Donabedian recomenda a tríade estrutura, processo e resultado. Estrutura refere-se aos instrumentos e recursos utilizados, assim como às condições físicas dos ambientes e organização dos serviços. Processo refere-se à execução do serviço e a relação entre profissional e usuário. A dimensão Resultados é o desfecho do serviço prestado e tem a satisfação como um de seus componentes ${ }^{3}$.

Já o modelo teórico de Andersen e Davidson ${ }^{4}$ coloca a satisfação do usuário com os serviços odontológicos como resultado de um processo dinâmico, em que cada variável que o compõe é importante, influenciando e sendo influenciada pelas demais. Nesse modelo, a satisfação do usuário é resultado de variáveis exógenas (grupos étnicos e corte etário); determinantes primários de saúde bucal (ambiente externo, saúde geral relatada, sistemas de atenção à saúde bucal e características pessoais), comportamentos de saúde bucal (práticas pessoais e uso formal de serviços) e condições de saúde bucal (condição de saúde bucal, percepção da condição de saúde bucal $)^{4}$. Assim, a satisfação com os serviços na perspectiva dos usuários ultrapassa o limite das características dos indivíduos, sofrendo influência dos contextos onde os serviços estão inseridos. É importante ressaltar que os usuários se sentem mais confortáveis para relatar insatisfação com serviços prestados fora do espaço assistencial ${ }^{5}$, entretanto não foram identificados estudos prévios que avaliaram a insatisfação com a assistência odontológica em inquéritos domiciliares que consideraram variáveis contextuais e individuais.

A insatisfação dos usuários com os serviços odontológicos abriga aspectos a serem desvelados, já que carrega particularidades dos contextos $^{6}$. Diante disso, este estudo avaliou, a partir de modelagem multinível, os fatores contextuais e individuais associados à insatisfação com os serviços odontológicos dos adultos brasileiros através do relato dessa insatisfação feito fora do espaço assistencial ${ }^{5}$, tendo como foco a melhoria do atendimento prestado.

\section{Métodos}

Estudo transversal multinível, uma vez que utilizou variáveis contextuais e individuais. A abordagem multinível utiliza o contexto para explicar o desfecho estudado em nível individual. Foram utilizados dados secundários, sendo que as variáveis contextuais foram obtidas em nível municipal, em diversas bases de dados públicas e as individuais foram coletadas nos domicílios dos participantes.

Em nível contextual, os dados foram coletados de bases de dados públicas e oficiais: do Censo Demográfico de 2010 do Instituto Brasileiro de Geografia e Estatística (IBGE) ${ }^{7}$; do Atlas Brasil, do Programa das Nações Unidas para o Desenvolvimento PNUD'; da Pesquisa Nacional de Saneamento Básico do IBGE do Data/SUS/MS 9 , que é um órgão pertencente à Secretaria Executiva do Ministério da Saúde, cujo funcionamento relaciona-se diretamente à ação produtora, receptora, ordenadora e disseminadora de informações. Atlas Brasil e a Pesquisa Nacional de Saneamento Básico são bases de dados geradas do censo demográfico de 2010, cuja coleta de dados foi realizada entre $1^{\circ}$ de agosto e 30 de outubro de 2010, utilizando a base territorial que se constituiu de 316574 setores censitários? ${ }^{7}$. Uma variável alusiva ao município, ser de capital ou interior, foi obtida do Projeto SBBrasil $2010^{10}$.

Em nível individual, foi utilizado o banco de dados da Pesquisa Nacional de Saúde Bucal, o Projeto SBBrasil 2010. A metodologia foi delineada a partir das recomendações propostas pela Organização Mundial de Saúde (OMS). Trata-se de uma amostra composta por 9779 indivíduos, representativa dos adultos (35-44 anos), oriunda de 177 municípios, incluindo as 26 capitais dos estados brasileiros e o Distrito Federal. A seleção dos municípios deu-se por amostragem probabilística por conglomerados, em múltiplos estágios com um efeito de desenho (deff) igual a 2 . Sortearam-se os municípios e setores censitários atendendo a técnica de probabilidade proporcional ao tamanho. Foram conduzidas entrevistas e exames intrabucais, sob luz natural, por cirurgiões-dentistas treinados e calibrados (Kappa $\geq$ 0,65), com auxílio de computadores de mão para registro das informações ${ }^{11}$. Apenas os adultos que utilizaram os serviços odontológicos alguma vez na vida e que responderam à pergunta relativa à variável dependente foram incluídos na análise. 


\section{Variável dependente}

A variável dependente "satisfação com os serviços odontológicos” foi obtida a partir da pergunta "Como você avaliaria a sua última consulta?". As opções de resposta "muito bom" e "bom" foram agrupadas no grupo dos satisfeitos e "regular", "ruim" e "péssimo" foram alocadas para o grupo dos insatisfeitos.

\section{Variáveis independentes}

As variáveis independentes seguiram o modelo teórico de Andersen e Davidson ${ }^{4}$, adaptado por Oliveira ${ }^{12}$, sendo: Variáveis de Contexto (ambiente, condição social/econômica, sistema de saúde, indicadores de assistência); Determinantes Primários Individuais (características pessoais imutáveis, e mutáveis); Comportamento (uso formal de serviços, motivo do uso, tipo de serviço utilizado); e Desfecho (condição de saúde bucal normativa/objetiva, condição de saúde bucal subjetiva, satisfação com os serviços odontológicos). Ressalta-se que um dos desfechos deste modelo é a variável dependente desse estudo.

\section{Variáveis independentes contextuais}

Quanto às Variáveis de Contexto, a organização no subgrupo ambiente foi: "localização geográfica do município" (capital; interior) ${ }^{10}$, "população com acesso ao banheiro e água encanada" (maior ou igual à média nacional; menor que a média nacional, de 90,81\%), "população com acesso à coleta de lixo" (maior ou igual à média nacional; menor que a média nacional, de 83,03\%) e "fluoretação da água distribuída" (sim; não $)^{7}$. O ponto de corte das variáveis "população com acesso ao banheiro e água encanada" e "população com acesso à coleta de lixo" foi obtido através da média das prevalências identificadas para cada município, considerando os municípios abordados neste estudo.

No subgrupo condição social/econômica as variáveis foram: índice de desenvolvimento humano municipal (IDHM) que é categorizado em muito baixo (de 0 a 0,49 ), baixo (entre 0,50 e 0,59), médio (entre 0,60 e 0,69), alto (entre 0,70 e 0,79 ) e muito alto (entre 0,80 e 1,0 ), esse indicador é um agregado de informações sobre renda, nível de instrução e longevidade em cada cidade $^{13}$, portanto optou-se categorizar no presente estudo em "alto/muito alto" e "muito baixo/baixo/médio”. O Coeficiente de Gini, que tem como objetivo mensurar a desigualdade na dis- tribuição de renda, a partir da curva de Lorenz, esse indicador varia entre 0 (completa igualdade) e 1 (completa desigualdade), e foi dicotomizada conforme a média nacional em 2010 (0,59), em maior desigualdade (menor ou igual a média) e menor desigualdade (maior que a média) ${ }^{7}$.

No subgrupo sistema de saúde as variáveis foram: financiamento per capita da população (discreta); proporção de dentistas por 1000 habitantes, proporção de dentistas no SUS por 1000 habitantes, cobertura de equipes de saúde bucal e presença de Centro de Especialidades Odontológicas no município. Essas informações foram coletadas no site DATA/SUS e na sala de gestão estratégica do Ministério da Saúde. O cálculo do número de dentistas é a razão entre o número de cirurgiões-dentistas, atuantes ou não no SUS, no município por 1000 habitantes do município, e foi categorizado em maior ou igual à média nacional e menor que a média nacional, sendo que a média para proporção de dentistas por 1000 habitantes foi de 0,66 e proporção de dentistas do SUS por 1000 habitantes foi de 0,41 e levou-se em conta apenas os municípios investigados. $\mathrm{O}$ cálculo da cobertura de equipes de saúde bucal na atenção primária foi agregado em maior ou igual à meta nacional e menor que a meta nacional, sendo que a meta para o ano de 2010 foi de $40,0 \%{ }^{14}$. A variável que informa a presença dos centros de especialidades nos municípios participantes do inquérito foi coletada considerando o ano 2010 e categorizada em presença e ausência.

No subgrupo indicadores de assistência as variáveis foram: média de escovação supervisionada no ano que foi dicotomizada em maior ou igual à meta nacional e menor que a meta nacional, sendo que a meta para o ano de 2010 foi de 3,0\% ${ }^{14}$.

\section{Variáveis independentes individuais}

No grupo Determinantes Primários Individuais foram incluídas no subgrupo características pessoais imutáveis as seguintes variáveis: sexo (feminino; masculino), idade em anos (35-39; 40-44) e cor da pele autodeclarada (brancos e amarelos/negros/pardos/indígenas). Para o subgrupo características pessoais mutáveis: anos de estudo (acima de 5 anos; analfabeto/entre 1 a 4 anos) e renda média familiar (maior ou igual à $\mathrm{R} \$ 1500,00$; menor que $\mathrm{R} \$ 1500,00)$.

No grupo Comportamento foram incluídas no subgrupo uso formal de serviços a seguinte variável: tempo desde a última consulta (há menos de 1 ano; há mais de um ano). No subgrupo tipo de serviço utilizado: (SUS; outros). 
Do grupo desfecho proposto no modelo de Andersen e Davidson ${ }^{4}$ foram incluídas as variáveis do grupo condição de saúde bucal no subgrupo normativa/objetiva: uso de prótese (não; sim) necessidade de prótese (não; sim) e dor de dente (não; sim). No subgrupo subjetiva: autopercepção da necessidade de tratamento (sim; não), autopercepção da necessidade de prótese (sim; não), satisfação com dentes e boca (satisfeitos; insatisfeitos) e o índice de impacto das desordens bucais no desempenho diário (OIDP) que foi calculado a partir da soma de todas as nove atividades diárias e foi dicotomizado em sem impacto (nenhuma atividade com impacto) e com impacto (uma ou mais atividade com impacto).

\section{Análise estatística}

Os dados referentes às variáveis contextuais e individuais foram organizados no software Statistical Package for the Social Sciences ${ }^{\oplus}$ (SPSS 19.0). Nas análises descritivas das variáveis individuais, considerou-se o efeito do desenho amostral complexo, fazendo uso do comando Complex Samples do referido software. Para as variáveis contextuais, utilizou-se o banco com dados dos municípios e não dos sujeitos para análise descritiva. Com o intuito de complementar as informações deste estudo, as proporções de insatisfação foram agrupadas pelas cinco macrorregiões geopolíticas brasileiras (Norte, Nordeste, Sul, Sudeste e Centro Oeste). Sendo assim, elaborou-se um mapa com a proporção de insatisfação nos municípios do interior divididas nas macrorregiões (desconsiderando a proporção nas capitais) e a proporção de insatisfação nas capitais. Utilizouse o software livre "Quantum Gis" que possibilita o georreferenciamento, edição e análise de dados.

Em um segundo momento realizou-se análises bivariadas (somente com as variáveis individuais), a partir do Teste Qui-quadrado. Somente as variáveis individuais que se mostraram relacionadas à variável dependente, considerando um nível de significância ( ) igual ou inferior a 0,20 , foram incluídas nas análises múltiplas.

Em um terceiro momento, os dados foram exportados para o programa STATA $^{\circledR} \mathrm{v} 14.0 \mathrm{com}$ o objetivo de determinar o modelo múltiplo multinível por meio de Regressão Logística Binária (utilizando dados contextuais e individuais), foi conduzida modelagem manual do tipo step backward. Foram mantidas no modelo final ajustado apenas as variáveis com $\mathrm{p} \leq 0,10$. Para verificação do ajuste do modelo, utilizou-se o pseudo $\mathrm{R}$ square $\left(\mathrm{R}^{2}\right)$ com o intuito de analisar a capacidade do modelo final ajustado em explicar a variação da variável dependente. A escolha do modelo final deu-se por meio do Log Likelihood, ou seja, o valor estimado do modelo vazio é multiplicado por (-2) e compara-se com o modelo pretendido, essa diferença deve ser a maior possível ${ }^{15}$.

Para estimação do ajuste entre desfecho (satisfação com os serviços odontológicos) e variáveis explicativas de primeiro (contextuais) e segundo (individuais) níveis de análise, utilizou-se o esquema de efeitos mistos e intersecção randômica $^{16}$. A priori considerou-se um modelo vazio (somente com intercepto aleatório e a variável dependente, sem as demais variáveis). Posteriormente, incluíram-se as variáveis do primeiro nível, seguidas pelas variáveis do segundo nível, com os coeficientes mistos com função logit para obtenção das medidas de odds ratios (OR) e IC 95\%. Observou-se redução na variabilidade do efeito aleatório, pela comparação com o modelo anterior. Compararam-se as funções de verossimilhança do modelo com e sem as variáveis da pessoa, usando o teste Qui-quadrado. Mantiveram-se no modelo somente as variáveis estatisticamente significantes $(\mathrm{p} \leq 0,10)$.

Obteve-se autorização para realização do Projeto SBBrasil 2010 através do Conselho Nacional de Ética em Pesquisa do Ministério da Saúde. Todos os participantes do Projeto SBBrasil 2010 assinaram o Termo de Consentimento, no qual foram esclarecidas as características do exame bucal a ser realizado, o sigilo dos dados obtidos e a livre decisão de participação do sujeito ${ }^{11}$.

\section{Resultados}

Dos 9.779 adultos entrevistados foram excluídos 836 por não terem utilizado os serviços odontológicos e/ou respondido à pergunta relacionada à variável dependente em análise, sendo assim, participaram desse estudo 8943 adultos, destes $14,9 \%(\mathrm{n}=1.426)$ mostraram-se insatisfeitos com os serviços odontológicos. A proporção de insatisfeitos com os serviços odontológicos nas capitais e nas cidades do interior, pelas regiões brasileiras, foram mais altas na Região Norte $(15,3 \%)$, e menores na região Sul (10,0\%) (Figura 1 ).

A análise descritiva das variáveis contextuais das bases de dados públicas relativa aos $177 \mathrm{mu}$ nicípios está apresentada na Tabela 1 e os dados descritivos individuais estão apresentados na Ta- 


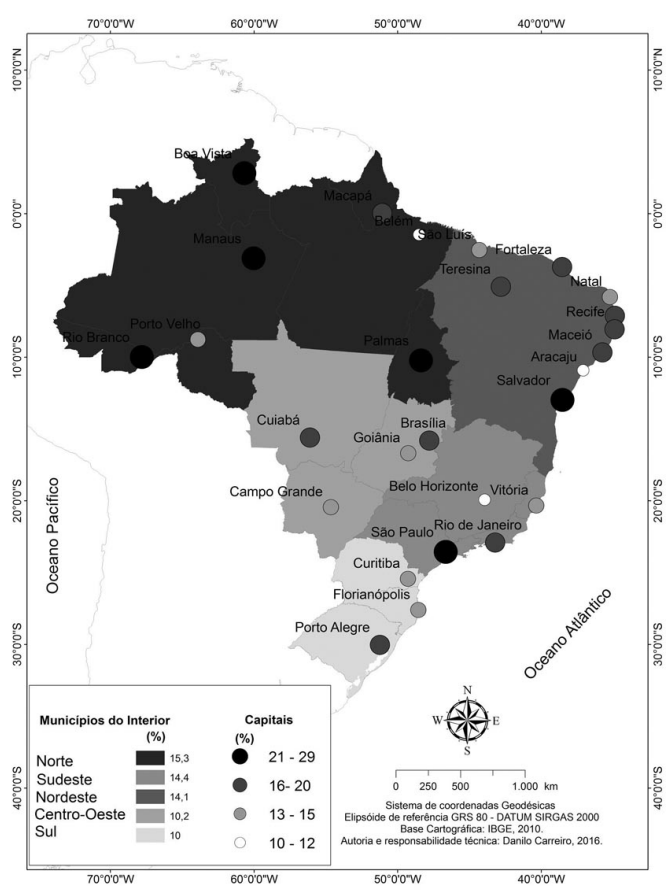

Figura 01. Proporção de indivíduos insatisfeitos com os serviços odontológicos utilizados, nas regiões brasileiras (municípios do interior) e nas capitais. Adultos participantes do Projeto SBBrasil 2010 $(\mathrm{n}=8943)$.

bela 2. Identificou-se predomínio dos que se autodeclararam brancos; com maior escolaridade; que usaram serviços por motivo de problemas bucais; que estavam insatisfeitos com dentes e boca e daqueles com impacto das desordens bucais no desempenho diário.

$\mathrm{Na}$ análise bivariada (Tabela 2) observou-se que a maioria das variáveis individuais mostramse associadas à insatisfação com os serviços odontológicos, exceto sexo, idade e uso de prótese.

No modelo múltiplo multinível ajustado, a chance de insatisfação com os serviços odontológicos foi maior para adultos que residiam em municípios com maior desigualdade social medida pelo coeficiente de Gini e com menor proporção de dentistas por habitante (variáveis contextuais). Entre as variáveis individuais, a insatisfação foi maior entre que se autodeclararam amarelos/negros/pardos/indígenas, entre os com menor escolaridade, os que consultaram-se por motivo de problemas bucais, entre os que estavam insatisfeitos com os dentes e boca e entre os que apresentaram impacto das desordens bucais no desempenho diário (Tabela 3).
Tabela 1. Análise descritiva das variáveis contextuais das bases de dados públicas $\mathrm{n}=177$ (municípios).

\begin{tabular}{|c|c|c|}
\hline VARIÁVEIS DE CONTEXTO & $\%$ & $\mathbf{n}$ \\
\hline \multicolumn{3}{|l|}{ Ambiente } \\
\hline \multicolumn{3}{|l|}{ Localização do município } \\
\hline Capital & 15,3 & 27 \\
\hline Interior & 84,7 & 150 \\
\hline \% da população com acesso à agua encanada & & \\
\hline Maior ou igual à média nacional & 52,0 & 92 \\
\hline
\end{tabular}

$48,0 \quad 85$

\% da população com acesso ao banheiro e água encanada

$\begin{array}{lrr}\text { Maior ou igual à média nacional } & 68,4 & 121 \\ \text { Menor que a média nacional } & 31,6 & 56\end{array}$

$\%$ da população com acesso à coleta de lixo

Maior ou igual à média nacional

$68,4 \quad 121$

Menor que a média nacional

$31,6 \quad 56$

Fluoretação da água distribuída

Sim

$66,7 \quad 118$

Não

$33,3 \quad 59$

Condição social/econômica

IDHM

Alto/muito alto

$55,4 \quad 98$

Muito baixo/baixo/médio

$44,6 \quad 79$

Coeficiente de Gini

Maior desigualdade

$74,0 \quad 131$

Menor desigualdade

$26,0 \quad 46$

Sistema de saúde

Financiamento per capita da população

Proporção dentistas por 1000 habitantes

Maior ou igual à média nacional

Menor que a média nacional

$45,2 \quad 80$

$54,8 \quad 97$

Proporção dentistas do SUS por 1000 habitantes

Maior ou igual à média nacional $\quad 34,5 \quad 61$

$\begin{array}{lll}\text { Menor que a média nacional } & 65,5 & 116\end{array}$

Cobertura equipes saúde bucal*

Maior ou igual à meta nacional $\quad 49,4 \quad 80$

$\begin{array}{lll}\text { Menor que a meta nacional } & 50,6 & 82\end{array}$

Presença de CEO

Presença

$44,1 \quad 78$

Ausência

$55,9 \quad 99$

Indicadores de assistência

Média escovação supervisionada*

Maior ou igual à meta nacional $\quad 25,5 \quad 40$

$\begin{array}{lll}\text { Menor que a meta nacional } & 74,5 & 117\end{array}$

Índice de cuidados odontológicos

$\begin{array}{lll}\text { Maior ou igual à média } & 49,2 & 87\end{array}$

Menor que a média $\quad 50,8 \quad 90$

*o n é menor que 177 municípios, dados não disponíveis. 
Tabela 2. Análise descritiva e bivariada dos fatores associados à insatisfação com os serviços odontológicos entre adultos, Projeto SB BRASIL, 2010, n=8943.

\begin{tabular}{|c|c|c|c|c|c|}
\hline VARIÁVEIS & n $\left(\%^{\star}\right)$ & Deff & Satisfeitos & Insatisfeitos & Valor $\mathbf{p}$ \\
\hline \multicolumn{6}{|c|}{ DETERMINANTES PRIMÁRIOS INDIVIDUAIS } \\
\hline \multicolumn{6}{|l|}{ Características pessoais imutáveis } \\
\hline \multicolumn{6}{|l|}{ Sexo } \\
\hline Feminino & $5923(62,9)$ & & 84,1 & 15,9 & \\
\hline Masculino & $3020(37,1)$ & 9,78 & 84,0 & 16,0 & 0,978 \\
\hline \multicolumn{6}{|l|}{ Idade (em anos) } \\
\hline De 35 a 39 anos & $4733(52,3)$ & & 84,1 & 15,9 & \\
\hline De 40 a 44 anos & $4210(47,7)$ & 6,77 & 84,0 & 16,0 & 0,831 \\
\hline \multicolumn{6}{|l|}{ Cor da pele ou raça autodeclarada } \\
\hline Brancos & $4681(58,9)$ & & 86,0 & 14,0 & \\
\hline Amarelos/Negros/Pardos/Indígenas & $4262(41,1)$ & 14,15 & 81,9 & 18,1 & 0,000 \\
\hline \multicolumn{6}{|l|}{ Características pessoais mutáveis } \\
\hline \multicolumn{6}{|l|}{ Anos de estudo ${ }^{* *}$} \\
\hline Acima de 5 anos & $7453(81,5)$ & & 84,4 & 15,6 & \\
\hline Analfabeto/ Entre 1 a 4 anos & $1447(18,5)$ & 14,88 & 81,8 & 18,2 & 0,013 \\
\hline \multicolumn{6}{|l|}{ Renda familiar ${ }^{\star \star}$} \\
\hline Maior ou igual à $\mathrm{R} \$ 1500,00$ & $3173(34,0)$ & & 87,4 & 12,6 & \\
\hline Menor que $\mathrm{R} \$ 1500,00$ & $5568(66,0)$ & 17,75 & 82,1 & 17,9 & 0,000 \\
\hline \multicolumn{6}{|l|}{ COMPORTAMENTO } \\
\hline \multicolumn{6}{|l|}{ Uso Formal dos Serviços } \\
\hline \multicolumn{6}{|l|}{ Tempo desde a última consulta ${ }^{\star *}$} \\
\hline Há menos de 1 ano & $4539(49,4)$ & & 86,5 & 13,5 & \\
\hline Há mais de 1 ano & $4323(50,6)$ & 7,59 & 81,5 & 18,5 & 0,000 \\
\hline \multicolumn{6}{|l|}{ Motivo do uso } \\
\hline \multicolumn{6}{|l|}{ Motivo da consulta ${ }^{\star *}$} \\
\hline Revisão/prevenção & $1958(21,3)$ & & 89,1 & 10,9 & \\
\hline Problemas bucais & $6975(78,3)$ & 7,91 & 82,7 & 17,3 & 0,000 \\
\hline \multicolumn{6}{|l|}{ Tipo de serviço utilizado } \\
\hline \multicolumn{6}{|l|}{ Tipo de serviço utilizado ${ }^{\star *}$} \\
\hline SUS & $5736(62,1)$ & & 86,1 & 13,9 & \\
\hline Outros & $3559(37,9)$ & 19,77 & 80,9 & 19,1 & 0,000 \\
\hline
\end{tabular}

\section{Discussão}

Esse estudo revelou que contextos de desigualdade social e menor relação dentista habitante apresentaram-se associados à maior insatisfação com os serviços odontológicos, o que merece destaque. Já no que tange às variáveis individuais, verificou-se maior chance de insatisfação entre os que se autodeclararam amarelos/negros/ pardos/indígenas, os com menor escolaridade, os que se consultaram por problemas bucais, os que estavam insatisfeitos com os dentes e boca e os que apresentaram impacto bucal no desempenho diário.
A prevalência de $14,9 \%$ de insatisfação com serviços odontológicos para adultos brasileiros registrada nesse estudo merece atenção, ao se considerar menores prevalências em pesquisas prévias conduzidas entre brasileiros: entre idosos $(3,7 \%)^{17} \mathrm{e}(9,5 \%)^{18}$; entre pessoas com idade igual ou superior a 18 anos $(9,0 \%)^{19}$; entre adultos da mesma faixa etária no Norte de Minas $(10,8 \%)^{6}$. Sugere-se que as diferenças encontradas podem ser explicadas pelas faixas etárias estudadas e/ou regiões em que foram desenvolvidos os respectivos estudos. Entre adultos brasileiros participantes do SBBrasil 2003, verificou-se prevalência similar à de $2010(14,3 \%)^{20}$. No panorama interna- 
Tabela 2. Análise descritiva e bivariada dos fatores associados à insatisfação com os serviços odontológicos entre adultos, Projeto SB BRASIL, 2010, $\mathrm{n}=8943$.

\begin{tabular}{|c|c|c|c|c|c|}
\hline VARIÁVEIS & $\mathbf{n}\left(\%^{*}\right)$ & Deff & Satisfeitos & Insatisfeitos & Valor $\mathrm{p}$ \\
\hline \multicolumn{6}{|c|}{ CONDIÇÃO DE SAÚDE BUCAL } \\
\hline \multicolumn{6}{|l|}{ Normativa/objetiva } \\
\hline \multicolumn{6}{|l|}{ Uso de prótese ${ }^{\star *}$} \\
\hline Não & $5611(65,6)$ & & 84,2 & 15,8 & \\
\hline Sim & $3150(34,4)$ & 8,02 & 83,7 & 16,3 & 0,558 \\
\hline \multicolumn{6}{|l|}{ Necessidade de prótese ${ }^{\star *}$} \\
\hline Não & $2175(31,4)$ & & 89,2 & 10,8 & \\
\hline Sim & $5879(68,6)$ & 12,90 & 82,0 & 18,0 & 0,000 \\
\hline \multicolumn{6}{|l|}{ Dor de dente ${ }^{\star *}$} \\
\hline Não & $6674(71,8)$ & & 86,1 & 13,9 & \\
\hline Sim & $2223(28,2)$ & 9,52 & 77,8 & 22,2 & 0,000 \\
\hline \multicolumn{6}{|l|}{ Subjetiva } \\
\hline \multicolumn{6}{|c|}{$\begin{array}{l}\text { Autopercepção da necessidade de } \\
\text { Tratamento }\end{array}$} \\
\hline Sim & $6963(77,2)$ & & 81,7 & 18,3 & \\
\hline Não & $1820(22,8)$ & 8,21 & 92,6 & 7,4 & 0,000 \\
\hline \multicolumn{6}{|c|}{$\begin{array}{l}\text { Autopercepção da Necessidade de } \\
\text { Prótese }^{\star \star}\end{array}$} \\
\hline Sim & $3548(36,3)$ & & 80,9 & 19,1 & \\
\hline Não & $5145(63,7)$ & 9,52 & 86,4 & 13,6 & 0,000 \\
\hline \multicolumn{6}{|l|}{ Satisfação com dentes e boca ${ }^{* *}$} \\
\hline Satisfeito & $3593(42,1)$ & & 92,1 & 7,9 & \\
\hline Insatisfeito & $5314(57,9)$ & 9,76 & 78,6 & 21,4 & 0,000 \\
\hline \multicolumn{6}{|l|}{ OIDP } \\
\hline Sem impacto & $4256(45,8)$ & & 88,9 & 11,1 & \\
\hline Com impacto & $4687(54,2)$ & 18,18 & 79,7 & 20,3 & 0,000 \\
\hline
\end{tabular}

${ }^{*}$ percentual com efeito de desenho. ${ }^{* *}$ número de respondentes menor que o tamanho amostral. ${ }^{* *}$ proporções aferidas para os municípios. n.a Não se aplica.

cional, verificaram-se prevalências de insatisfação de $11 \%$ entre adultos do Reino Unido ${ }^{21} ; 11 \%$ na África do $\mathrm{Sul}^{22}$ e $19 \%$ entre adultos, por inquérito telefônico, na China ${ }^{23}$. Altas proporções de satisfação com os serviços odontológicos podem ser fruto do fenômeno conhecido como efeito de "elevação" das taxas de satisfação, reportado mesmo quando as experiências são negativas ${ }^{18}$.

A insatisfação com os serviços odontológicos apresentou distribuição que variou com o nível de desenvolvimento das regiões brasileiras, com menores proporções de insatisfação registradas nas regiões mais desenvolvidas: $10,0 \%$ no Sul e $10,2 \%$ no Centro-Oeste. Inversamente, as maiores proporções de insatisfação foram identificadas nas regiões menos desenvolvidas: $15,3 \%$ no Norte e $14,1 \%$ no Nordeste. Chama atenção a proporção de insatisfação entre residentes da região Sudeste, que apesar de contar com estados desenvolvidos, teve proporção de $14,4 \%$ de insatisfação. Apesar de ter encontrado associação com índice de Gini e insatisfação, quando verificada a insatisfação nos municípios do interior, verifica-se variações entre regiões menos e mais desenvolvidas.

Identificou-se maior chance de insatisfação com os serviços odontológicos entre adultos residentes em municípios com maior desigualdade social, pesquisa multinível antecedente, entre usuários do SUS de 62 municípios brasileiros, identificou que pessoas de municípios com menor renda per capita estavam mais insatisfeitas com os serviços de saúde ${ }^{24}$. Resultado esse que contradiz pesquisa prévia, que ao abordar considerações teórico-conceituais acerca de 56 estudos publicados na literatura nacional sobre satisfação, identificou associação entre maior grau de pobreza e maior satisfação ${ }^{25}$. Por outro lado, pesquisa anterior que utilizou dados da Pesquisa $\mathrm{Na}$ cional por Amostra de Domicílios (PNAD) 2003 


\begin{tabular}{|c|c|c|c|c|}
\hline \multirow[t]{33}{*}{ 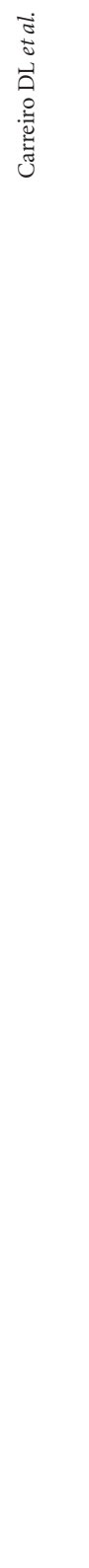 } & $\begin{array}{l}\text { Tabela 3. Análise múltipla multinível dos fat } \\
\text { entre adultos, Projeto SB BRASIL, 2010, } \mathrm{n}=8943 \text {. }\end{array}$ & isfação & os serviços & tológicos \\
\hline & VARIÁVEIS & OR & IC $^{95 \%}$ & Valor p \\
\hline & VARIÁVEIS DE CONTEXTO & & & \\
\hline & Condição social /econômica & & & \\
\hline & Coeficiente de Gini & & & \\
\hline & Menor desigualdade & Ref. & & \\
\hline & Maior desigualdade & 1,53 & $1,31-1,81$ & 0,000 \\
\hline & Sistema de saúde & & & \\
\hline & Proporção dentistas por 1000 habitantes & & & \\
\hline & Maior ou igual à média nacional & Ref. & & \\
\hline & Menor que a média nacional & 1,17 & $1,00-1,37$ & 0,053 \\
\hline & DETERMINANTES PRIMÁRIOS INDIVIDUAIS & & & \\
\hline & Características pessoais imutáveis & & & \\
\hline & Cor da pele ou raça autodeclarada & & & \\
\hline & Brancos & Ref. & & \\
\hline & Amarelos/Negros/Pardos/Indígenas & 1,12 & $0,99-1,27$ & 0,078 \\
\hline & Características pessoais mutáveis & & & \\
\hline & Anos de estudo & & & \\
\hline & Acima de 5 anos & Ref. & & \\
\hline & Analfabeto/ Entre 1 a 4 anos & 1,14 & $0,98-1,33$ & 0,097 \\
\hline & COMPORTAMENTO & & & \\
\hline & Motivo do uso & & & \\
\hline & Motivo da consulta & & & \\
\hline & Revisão/prevenção & Ref. & & \\
\hline & Problemas bucais & 1,23 & $1,04-1,44$ & 0,015 \\
\hline & CONDIÇÃO DE SAÚDE BUCAL & & & \\
\hline & Subjetiva & & & \\
\hline & Satisfação com dentes e boca & & & \\
\hline & Satisfeito & Ref. & & \\
\hline & Insatisfeito & 2,60 & $2,25-3,02$ & 0,000 \\
\hline & OIDP & & & \\
\hline & Sem impacto & Ref. & & \\
\hline & Com impacto & 1,48 & $1,30-1,69$ & 0,000 \\
\hline
\end{tabular}

Pseudo R Square $=8,0 \%$; Log Likelihood do modelo vazio=-3891,37; Log Likelihood do modelo cheio=-3670,77.

e 2008, identificou que pessoas com melhores condições econômicas avaliaram o serviço odontológico como "muito/bom" e "bom", tanto em 2003 quanto em $2008^{26}$. Apesar do índice de Gini não medir renda e sim desigualdade de renda, verifica-se que no Brasil ocorreu queda significativa deste indicador na última década. Esse fenômeno pode ser explicado pela redução do desemprego, aliada ao aumento de rendimento dos trabalhadores e à política de incentivo à formalização ${ }^{7}$.

Averiguou-se maior chance de insatisfação com os serviços odontológicos entre adultos residentes em municípios com menor proporção de dentistas/habitantes, mesmo após controle pelas demais variáveis. A menor proporção de dentista na população, sugere menor oferta de assistência odontológica, o que pode resultar em menor acesso. A dificuldade de acesso pode gerar maior insatisfação na população como um todo. No Brasil, a oferta dos serviços é ainda bastante heterogênea, com maior concentração de profissionais nas regiões mais ricas ${ }^{11}$, atendendo mais as leis de mercado do que ao perfil das necessidades. Verificou-se, nesse estudo, que a proporção de dentistas por habitante foi significativamente menor em regiões em que a insatisfação foi maior (dados não mostrados), no caso Norte (20,1\% de insatisfação e 0,38 dentistas/1000habitantes) e Nordeste (16,5\% de insatisfação e 0,55 dentistas/1000habitantes), quando comparados 
com as regiões Sul (11,7\% de insatisfação e 0,87 dentistas/1000habitantes) e Sudeste (15,5\% de insatisfação e 0,90 dentistas/1000habitantes). A organização e ordenação dos recursos deve proporcionar oferta equânime do serviço pelo país, uma vez que apesar de ter uma grande proporção de dentistas no país, esses profissionais estão concentrados em regiões como Sul e Sudeste ${ }^{11}$. Assim, espera-se que a oferta de serviços odontológicos siga o perfil das necessidades da população, o que contribuirá com maior equidade na oferta de assistência à saúde bucal e, possivelmente, com o aumento das proporções de indivíduos satisfeitos com tais serviços.

Quanto aos determinantes primários individuais, esse estudo identificou que adultos que se autodeclararam amarelos/negros/pardos/indígena tiveram chance $12 \%$ maior de estarem insatisfeitos com os serviços odontológicos enquanto adultos menos escolarizados tiveram chance $14 \%$ maior. Devido à grande exclusão social ocorrida historicamente, pretos e pardos apresentam indicadores sociais desfavoráveis, o que gera diferenças significativas no acesso aos direitos básicos de educação e saúde, menor utilização dos serviços de saúde bucal ${ }^{27}$, além de piores condições normativas de saúde bucal já evidenciadas na literatura ${ }^{28}$. Dados da Pesquisa Mundial de Saúde (2003), conduzida em 21 países Europeus, identificou correlação positiva entre escolaridade e insatisfação ${ }^{29}$. Por outro lado, estudo prévio conduzido entre idosos participantes do Projeto SBBrasil 2003, identificou associação entre insatisfação e maior escolaridade ${ }^{17}$, resultado esse que diverge ao encontrado no presente estudo. Possivelmente essa situação seja mais frequente entre idosos do que entre adultos. Talvez menor grau de escolaridade pode gerar maior dificuldade no entendimento dos fatores relacionados ao serviço utilizado. Também pode haver falta de entendimento do tratamento realizado, bem como comunicação difícil entre usuário e profissional, que por sua vez, podem gerar maior insatisfação ${ }^{19}$. Resultado similar ao do presente estudo foi encontrado em uma pesquisa de base populacional, com amostra 781 usuários maiores de idade do interior de Minas Gerais, em que a insatisfação esteve associada à menor escolaridade ${ }^{19}$.

Quanto ao comportamento de saúde bucal, identificou-se que pessoas que apresentaram como motivo da consulta problemas bucais tiveram chance $23 \%$ de se mostrarem insatisfeitas. A educação em saúde é uma das estratégias importantes na prevenção de doenças, nesse sentido, o prestador de assistência tem papel fundamental em orientar o usuário sobre como evitar problemas bucais. Pesquisa prévia identificou a associação entre não receber informações sobre como evitar problemas bucais e insatisfação com os serviços odontológicos ${ }^{6,17}$. Assim como satisfação com os serviços odontológicos e receber informações sobre como evitar problemas bucais ${ }^{18}$.

Quanto às condições subjetivas de saúde bucal, identificou-se que pessoas insatisfeitas com os dentes e boca $(\mathrm{OR}=2,3)$ e que relataram impacto das desordens bucais no desempenho diário $(\mathrm{OR}=1,48)$ apresentaram maiores chances de insatisfação com os serviços odontológicos. Já foi observado que questões subjetivas, quando percebidas negativamente, têm impacto na insatisfação com os serviços odontológicos entre adultos ${ }^{6}$ e entre idosos ${ }^{6,17,19}$. A maior insatisfação, entre os adultos que apresentaram percepção negativa de sua condição bucal, revela a importância da visão dos usuários sobre sua própria saúde no momento em que avaliam a qualidade dos serviços de saúde utilizados ${ }^{2,4}$. É interessante observar que a avaliação dos serviços foi afetada por variáveis de autopercepção, mas não por variáveis normativas de saúde bucal. Resultado semelhante já foi verificado na literatura ${ }^{6}$.

Convém comentar ainda, que no modelo múltiplo final, o tipo de serviços utilizado (SUS versus outros) não se revelou associado à insatisfação com os serviços odontológicos e pode evidenciar que os serviços públicos estão em consonância com os privados em termos da satisfação alcançada. Este achado já havia sido observado ${ }^{6}$. Entre idosos, outro estudo verificou maior satisfação com serviços odontológicos entre usuários do SUS ${ }^{18}$. Esses achados podem indicar efeitos positivos das estratégicas públicas de assistência em saúde bucal criadas nos últimos anos através do Brasil Sorridente.

Ressalta-se que o processo da avaliação de serviços odontológicos na perspectiva dos usuários e as variáveis pesquisadas é mutável, portanto, causas e efeitos certamente variam ao longo da prestação de serviço que é dada e as distintas políticas envolvidas. Portanto, sendo este um estudo seccional, que não permite estabelecer a relação temporal entre as associações observadas, a interpretação dos achados é limitada. Por outro lado, destaca-se o fato de se tratar de um estudo da população adulta brasileira, o que reflete o estado dessa população e também pode refletir o impacto das políticas de saúde bucal, preconizadas pela OMS que o Brasil vem desenvolvendo. Ademais, poderá nortear para previsões de necessidades futuras para os idosos brasileiros. Um 
destaque do presente estudo é a análise multinível, que utilizou dados contextuais para explicação de um desfecho de saúde bucal e sua interação com variáveis individuais.

\section{Conclusão}

A implementação ou adequação de políticas públicas com o intuito de melhorar a satisfação com os serviços odontológicos deve priorizar os municípios com maior desigualdade social e aqueles carentes de dentistas. Concomitantemente, é necessário que tais políticas que possam priorizar os socialmente desfavorecidos; bem como aqueles que autopercebem problemas bucais; aqueles que se encontram insatisfeitos com sua saúde bucal; e aqueles com impactos decorrentes dos problemas bucais. A oferta dos serviços de saúde bucal é ainda heterogênea no país, com maior concentração de profissionais nas regiões mais ricas (Sul e Sudeste). É necessário que a distribuição dos serviços de saúde bucal no país siga as demandas da população, contribuindo no alcance de maior equidade na oferta e uso dos serviços de saúde bucal e, consequentemente, atingindo aumento das proporções de indivíduos satisfeitos com tais serviços.

\section{Colaboradores}

Concepção e delineamento: DS Haikal; AMEBL Martins; DL Carreiro. Coleta de Dados contextuais: DL Carreiro; RFR Oliveira. Análise e interpretação dos dados: DS Haikal; DL Carreiro. Redação: DL Carreiro; AMEBL Martins; RFR Oliveira; WLM Coutinho; DS Haikal. Revisão crítica e Aprovação final da versão a ser publicada: DS Haikal; AMEBL Martins.

\section{Agradecimentos}

Danilo Lima Carreiro é bolsista de doutorado da Capes. Andréa Maria Eleutério de Barros Lima Martins é bolsista de Pós Doutorado Júnior do CNPQ. Desirée Sant Ana Haikal é bolsista de produtividade da Fapemig. 


\section{Referências}

1. Lorena Sobrinho JE, Martelli PJL, Albuquerque MS, Lyra TM, Farias SF. Acesso e qualidade: avaliação das equipes de saúde bucal participantes do PMAQ-AB 2012 em Pernambuco. Saúde Debate 2015; 39(104):136-46.

2. Martins AMEBL, Barreto SM, Pordeus IA. Uso de serviços odontológicos entre idosos brasileiros. $\mathrm{Rev} \mathrm{Pa}$ nam Salud Publica 2007; 22(5):308-316.

3. Donabedian A. The quality of care: how can it be assessed? JAMA 1988; 260(12):1743-1748.

4. Andersen RM, Davidson PL. Ethnicity, aging, and oral health outcomes: a conceptual framework. Adv Dent Res 1997; 11(2):203-209.

5. Silva Filho JF, Cavalcanti MT, Cadilhe GR, Vilaça AT, Avancini E, Machado HO, Perecmanis L, Maisonnette MJ, Rocha EC, Carreteiro RO, Mozinho GMP, Pitta AM. Avaliação de qualidade de serviços de saúde mental no Município de Niterói - RJ. A satisfação do usuário como critério avaliador. J Bras Psiquiat 1996; 45(7):393-402.

6. Roberto LL, Martins AMEBL, De Paula AMB, Ferreira EF, Haikal DS. Insatisfação com os serviços odontológicos e fatores associados entre adultos. Cien Saude Colet 2015; 22(5):1601-1613.

7. Instituto Brasileiro de Geografia e Estatística (IBGE). Síntese de indicadores sociais: uma análise das condições de vida da população brasileira. Estudos e Pesquisas 2011; 27:1-4.

8. Atlas do Desenvolvimento Humano no Brasil. 2010. [acessado 2016 Jun 06]. Disponível em: http://www. atlasbrasil.org.br/2013/pt/consulta

9. Brasil. Ministério da Saúde (MS). Informações de saúde Informação de saúde: cadernos de informação de saúde. 2010. [acessado 2016 Jun 06]. Disponível em: http:// tabnet.datasus.gov.br/tabdata/cadernos/cadernosmap. htm

10. Brasil. Ministério da Saúde (MS). Departamento de Atenção Básica. Sb Brasil 2010. Pesquisa nacional de saúde bucal: Resultados principais. Brasília: MS; 2011.

11. Roncalli AG, Silva NND, Nascimento AC, Freitas CHSDM, Casotti E, Peres KG, Moura L, Peres MA, Freire MCM, Cortes MIS, Vettore MV, Paludetto Júnior M, Figueiredo N, Goes PSA, Pinto RS, Marques RAA, Moysés SJ, Reis SCGB, Narvai PC. Aspectos metodológicos do Projeto SBBrasil 2010 de interesse para inquéritos nacionais de saúde. Cad Saude Publica 2012; 28(Supl.):40-57.

12. Oliveira RFR. Desigualdades sociais no uso de serviços odontológicos: comparação entre a assistência prestada pelo SUS e outros serviços [tese]. Montes Claros: Universidade Estadual de Montes Claros; 2016.

13. Frias AC, Antunes JLF, Junqueira SR, Narvai PC. Determinantes individuais e contextuais da prevalência de cárie dentária não tratada no Brasil. Rev Panam Salud Publica 2007; 22(4):279-285.

14. Brasil. Portaria no 3.840 , de 7 de dezembro de 2010. Inclui a Saúde Bucal no Monitoramento e Avaliação do Pacto pela Saúde e estabelece as diretrizes, orientações e prazos do processo de ajuste de metas para o ano de 2011. Diário Oficial da União 2010; $8 \mathrm{dez}$.
15. Carle AC. Fitting multilevel models in complex survey data with design weights: Recommendations. $B M C$ Medical 2009; 9:49.

16. Snijders TAB. Multilevel analysis. Berlin Heidelberg: Springer; 2011.

17. Martins AMEBL, Jardim LA, Souza JGS, Rodrigues CAQ, Ferreira RC, Pordeus IA. A avaliação negativa dos serviços odontológicos entre idosos brasileiros está associada ao tipo de serviço utilizado? Rev Bras Epidemiologia 2014; 17(5):71-90.

18. Rodrigues CAQ, Silva PLV, Caldeira AP, Pordeus IA, Ferreira RC, Martins AMEB. Fatores associados à satisfação com serviços odontológicos entre idosos. Rev Saude Publica 2012; 46(6):1039-1050.

19. Martins AMEBL, Ferreira RC, Santos-Neto PE, Carreiro DL, Souza JGS, Ferreira EF. Insatisfação dos usuários com a assistência odontológica: estudo domiciliar populacional. Rev Saude Publica 2015; 49:51.

20. Brasil. Ministério da Saúde (MS). Projeto SB Brasil 2003: condições de saúde bucal da população brasileira 2002-2003: resultados principais. Brasília: MS; 2004.

21. Bedi R, Gulati N, McGrath C. A study of satisfaction with dental services among adults in the United Kingdom. Br Dent J. 2005; 198(7):433-437.

22. Myburgh NG, Solanki GC, Smith MJ, Lalloo R. Patient satisfaction with health care providers in South Africa: the influences of race and socioeconomic status. Int $\mathrm{J}$ Qual Health Care 2005; 17(6):473-477.

23. Tung YC, Chang GM. Patient satisfaction with and recommendation of a primary care provider: associations of perceived quality and patient education. Int J Qual Health Care 2009; 21(3):206-213.

24. Passero LG. Insatisfação do usuário da atenção básica com o SUS - análise multinível da pesquisa da ouvidoria [dissertação]. Porto Alegre: Universidade Federal do Rio Grande do Sul; 2013.

25. Esperidião MA, Trad LAB. Avaliação da satisfação dos usuários: considerações teórico-conceituais. Cad Saude Publica 2006; 22(6):1267-1276.

26. Peres KG, Peres MA, Boing AR, Berdoldi AD, Bastos JL, Barros AJD. Redução das desigualdades sociais na utilização de serviços odontológicos no Brasil entre 1998 e 2008. Rev Saude Publica 2012; 46(2):250-258.

27. Souza HAS, Oliveira PAP, Paegle AC, Goes PSA. Raça e uso de serviços de saúde buccal por idosos. 2012; 17(8):2063-2070.

28. Guiotoku SK, Moysés ST, Moysés SJ, França BHS, Bisnelli JC. Iniquidades raciais em saúde bucal. Rev Panam Salud Publica 2012; 31(2):135-141.

29. Bleich SN, Ozaltin E, Murray CJL. How does satisfaction with the health-care system relate to patient experience? Bull World Health Organ 2009; 87(4):271-278.

Artigo apresentado em 05/06/2016

Aprovado em 26/01/2017

Versão final apresentada em 28/01/2017 
